\title{
Association between the levels of CGI-58 and lipoprotein lipase in the placenta of patients with preeclampsia
}

\author{
JIANXIN DONG, MIAO WANG, JIE GAO, JIE LIU and YAN CHEN \\ Department of Obstetrics and Gynecology, North China University of Science and \\ Technology Affiliated Hospital, Tangshan, Hebei 063000, P.R. China
}

Received September 16, 2020; Accepted June 25, 2021

DOI: $10.3892 /$ etm.2021.10563

\begin{abstract}
Preeclampsia is an idiopathic disease of pregnancy, which seriously endangers the life of both the mother and the infant. The pathogenesis of preeclampsia has not been fully elucidated, although it is generally considered to be associated with abnormal lipid metabolism during pregnancy. Comparative gene identification-58 (CGI-58) and lipoprotein lipase (LPL) are involved in the first step of triglyceride hydrolysis and serve an important role in lipid transport in the placenta. The present study aimed therefore to investigate the association between CGI-58 and LPL in the placentas of patients with or without preeclampsia and to evaluate blood lipid levels. The patient cohort was divided into two groups, pregnant women with preeclampsia and normal pregnant women (control). According to biochemical analyses, reverse transcription-quantitative PCR, immunohistochemistry analysis and western blotting, the expression of CGI-58 and LPL in the placenta was detected, the blood lipid levels were evaluated and other clinical data were collected. Compared with the control group, triglycerides (TGs), low density lipoprotein-cholesterol (LDL-C), apolipoprotein B (ApoB) and atherosclerotic index (AI) were significantly higher in the preeclampsia group, whereas high density lipoproteincholesterol (HDL-C) and apolipoprotein A (ApoA) were significantly lower $(\mathrm{P}<0.05)$. Furthermore, the expression levels of CGI-58 and LPL in the placental tissue of the preeclampsia
\end{abstract}

Correspondence to: Professor Yan Chen, Department of Obstetrics and Gynecology, North China University of Science and Technology Affiliated Hospital, 73 Jianshe Road, Tangshan, Hebei 063000, P.R. China

E-mail: yanchen0806@yahoo.com

Abbreviations: AI, atherosclerotic index; ApoA, apolipoprotein A; ApoB, apolipoprotein B; ATGL, adipose triglyceride lipase; CGI-58, comparative gene identification-58; HDL-C, high density lipoprotein cholesterol; LDL-C, low density lipoprotein cholesterol; Lp(a), lipoprotein small a; LPL, lipoprotein lipase; oxLDL, oxidized low-density lipoprotein; TC, total cholesterol; TG, triglyceride

Key words: preeclampsia, comparative gene identification-58, lipoprotein lipase, placenta group was significantly lower than that of the control group $(\mathrm{P}<0.05)$. Linear correlation analysis demonstrated that there was a positive association between CGI-58 and LPL ( $\mathrm{r}=0.602$; $\mathrm{P}<0.05)$, that CGI-58 was positively associated with HDL-C $(\mathrm{r}=0.63 ; \mathrm{P}<0.01)$ but negatively associated with TG and ApoB ( $r=0.840 ; \mathrm{P}<0.01$; and $\mathrm{r}=0.514 ; \mathrm{P}<0.05$, respectively), that LPL was positively associated with HDL-C $(\mathrm{r}=0.524 ; \mathrm{P}<0.01)$ but negatively associated with TG and $\mathrm{AI}(\mathrm{r}=0.659 ; \mathrm{P}<0.01$; and $\mathrm{r}=0.496 ; \mathrm{P}<0.01$, respectively). These results suggested that the expression of CGI-58 and LPL in the placenta was associated with the pathogenesis of preeclampsia and maternal lipids and the risk of preeclampsia was increased with decreasing expression levels of CGI-58 and LPL. Hence, CGI-58 and LPL may be used as important indicators for the diagnosis of preeclampsia and for the prevention of preeclampsia in pregnant women.

\section{Introduction}

Preeclampsia is a pregnancy-specific clinical syndrome with an incidence rate of $3-6 \%$, which is responsible for $10-15 \%$ of maternal deaths $(1,2)$. The classical definition of preeclampsia is a novel-onset hypertension combined with novel-onset proteinuria after gestational week 20, or a novel-onset preeclampsia-associated signs without proteinuria (3). Preeclampsia can cause maternal pulmonary edema, abnormal expression of liver enzymes, eclampsia and central nervous system complications, but can also cause fetal growth and development-related complications (4). The clinical characteristics of preeclampsia demonstrate notable heterogeneity, suggesting that it might be difficult to explain the pathogenesis of preeclampsia based on a single etiological factor (5). At present, preeclampsia is generally considered to involve multiple factors, pathways and mechanisms (6). Its etiology may be closely associated with insufficient invasion of trophoblasts, increased oxidative stress, abnormal immune regulation mechanisms, vascular endothelial damage and genetic mechanisms (7). However, there is a consensus that placental dysfunction serves a crucial role in the development of preeclampsia (8).

The placenta is the foundation of the maternal-infant relationship, and maternal-fetal nutrient transport is the basis of fetal growth and development (9). Lipid transport in the placenta is not only the primary source of fetal energy supply, but also the key material that promotes the development of the 
fetal nervous system $(10,11)$. Lipid metabolism disorder can cause inflammatory reactions and accumulation of oxidative stress products, affecting trophoblast invasion and placental development and causing vascular endothelial damage and inhibition, leading to a series of pathological changes (12). Compared with normal pregnant women, the serum lipid levels and lipoprotein metabolism of patients with preeclampsia is abnormal (13). High blood lipid levels in patients with preeclampsia lead to atherosclerotic changes during pregnancy, which can persist for several years after delivery, increasing the risk of cardiovascular disease (14). This may be due to the increase in blood vessel injury components, such as serum C-reactive protein, homocysteine and dimethylarginine and a decrease in vascular protective components, such as vascular endothelial growth factor and angiopoietins 2 in patients with preeclampsia (15). Lipid metabolism disorders might therefore lead to the production of a large number of lipid peroxides, resulting in vasospasm and endothelial cell damage (16).

Placental lipid transport is an important energy source for the fetus. Maternal triglycerides (TGs) need to be decomposed into fatty acids by the adipose TG lipase (ATGL) of the placenta before entering the fetus (17). Lipoprotein lipase (LPL) is a member of the ATGL family that is widely present in adipocytes, macrophages, myocardium and skeletal muscle (18). LPL is involved in the hydrolysis of TGs in chylomicrons and very low-density lipoprotein granules, and their products can be used for oxidative energy supply and lipid metabolism (18). The disorder of placental lipid transport can lead to lipid accumulation and increased lipid peroxidation with oxidative activity, resulting in inflammation, inward flow of extravascular lipids, and a further increase in vascular permeability, resulting in endothelial damage (19). Resistin can upregulate the expression of LPL in macrophages via the PPAR $\gamma$-dependent PI3K/Akt signaling pathway, thus accelerating the transfer of extracellular oxidized low-density lipoprotein (oxLDL) to macrophages (20). The increase of LPL expression not only contributes to the hydrolysis of intracellular TG, but also promotes the accumulation of lipids in macrophages (20).

Comparative gene identification-58 (CGI-58) is a glycoprotein with a molecular weight of $28 \mathrm{~kb}$, which is highly expressed in fat, liver, testis, muscle and other tissues, and can be used as an agonist in the hydrolysis of ATGL and lysophosphatidic acid acyltransferase $(21,22)$. Due to co-activation, LPL can increase its autocatalytic activity by 20 times by binding with co-activating protein CGI-58 (13). Under the influence of maternal obesity, dyslipidemia and elevated insulin levels, CGI-58 is involved in the regulation of the TG hydrolysis pathway in fetal placenta. CGI-58 knockout macrophages are similar to foam cells, which contain large amounts of triglycerides and cholesteryl esters (23). Triglycerides and cholesteryl esters are easy to deposit under vascular endothelial cells and cause oxidative stress due to defective PPAR $\gamma$ signaling (24). Under the stimulation of oxLDL, the deposition of lipid droplets in macrophages is increased, which aggravates the formation of atherosclerosis (25). Lipid metabolism disorder can cause hyperlipidemia and accumulation of lipolysis products, which might cause endothelial cell damage, such as vasoconstriction and hypercoagulability, which are related to the pathogenesis of a preeclampsia (26). The oxidative stress caused by lipid metabolism disorder in the placenta is enhanced, and the invasive ability as well as apoptosis in the trophoblasts is decreased, which affect the lipid transport function of the placenta (27). CGI-58 and LPL are therefore involved in the process of placental TG hydrolysis; however, whether both are related to preeclampsia requires further investigation.

In the present study, the expression of CGI-58 and LPL in the placenta and the blood lipid levels were determined. The association between CGI-58 and LPL in the placenta with preeclampsia and blood lipid levels were further examined. The results from the present study may improve the current understanding of the pathogenesis of preeclampsia and highlight novel treatment strategies for patients.

\section{Materials and methods}

Patients. A total of 37 pregnant women with preeclampsia and 40 normal pregnant women in the Department of Obstetrics and Gynecology of the North China University of Science and Technology Affiliated Hospital (Tangshan, China) were recruited between September 2018 and July 2019. The present study was approved by the Ethics Committee of the North China University of Science and Technology Affiliated Hospital (approval no. 20180718A) and performed in accordance with the Declaration of Helsinki. All participants provided written informed consent for the use of their placenta, blood and clinical information.

The diagnostic criteria of preeclampsia were as follows: After 20 weeks of gestation, systolic blood pressure was $\geq 140 \mathrm{mmHg}$ and/or diastolic blood pressure was $\geq 90 \mathrm{mmHg}$, with proteinuria $\geq 0.3 \mathrm{~g} / 24 \mathrm{~h}$, or random urine protein $(+)$; or, if there was no proteinuria, any of the following: i) thrombocytopenia (platelet $<100 \times 10^{9} / 1$ ); ii) liver function damage (serum transaminase level $>2$ times that of normal values; iii) impairment of renal function (serum creatinine levels $>1.1 \mathrm{mg} / \mathrm{dl}$ or $2 \mathrm{x}$ the normal value); iv) pulmonary edema; or v) novel central nervous system abnormalities or visual disorders (3). The inclusion criteria for the preeclampsia group were as follows: Diagnosis of preeclampsia, gestational weeks between 37 and 41 and singleton birth by cesarean section. Were excluded from the preeclampsia group pregnant women with the following diseases: Chronic hypertension, diabetes mellitus, gestational diabetes mellitus, thyroid dysfunction, nephritis, cholestasis syndrome and autoimmune diseases, severe infectious diseases or premature rupture of membranes during pregnancy. The inclusion criteria for the control group were as follows: Healthy pregnant women who gave birth by cesarean section due to social factors, scarred uterus or cephalopelvic disproportion, no history of hypertension, singleton delivery and gestational weeks between 37 and 41 . The exclusion criteria for the control group was pregnant woman who did not provide consent.

Placental tissue collection. Within 30 min of delivery, two placental tissue sections of $\sim 1 \mathrm{~cm}^{3}$ were randomly taken from the maternal surface of the placenta under strict aseptic conditions. Embolic and calcified sites were avoided. One sample was stored at $-80^{\circ} \mathrm{C}$ for western blotting and RT-qPCR analysis. The other sample was fixed using $10 \%$ formalin for $24 \mathrm{~h}$ at room temperature, embedded in paraffin and sectioned with thickness of $\sim 3 \mathrm{~mm}$ for immunohistochemical analysis. 
Biochemical analyses. Fasting venous blood (fasting for $\geq 8 \mathrm{~h}$ ) was collected 1 week before delivery, and the blood lipid levels were measured. Based on cholesterol oxidase method, total cholesterol (TC) was determined with the total cholesterol test kit (cat. no. OSR6116; Beckman Coulter) according to the manufacturers' instructions. TG level was evaluated by GPO-PAP method (cat. no. GS111Z), high density lipoprotein cholesterol (HDL-C) was evaluated by selective inhibition method (cat. no. GS131Z), low density lipoprotein cholesterol (LDL-C) was evaluated by surfactant removal method (cat. no. GS141Z), lipoprotein small a [Lp(a)] was evaluated by immunological turbidimetry assay (cat. no. GS151Z), apolipoprotein A (ApoA) was evaluated by immunological turbidimetry assay (cat. no. GS161Z), and apolipoprotein B (ApoB) was evaluated by immunological turbidimetry assay (cat. no. GS171Z). All these kits were purchased from Beijing Jiuqiang Biotechnology Co., Ltd. Routine blood tests were performed by the Laboratory Department of North China University of Science and Technology Affiliated Hospital using AU5800 Chemistry Analyzer (Beckman Coulter). The Department of Obstetrics and Gynecology collected the clinical examination data, including age, gestational week, number of pregnancies, number of births, uterine height, abdominal circumference, neonatal weight, BMI before pregnancy and gestational weight gain.

Reverse transcription-quantitative PCR (RT-qPCR). Total RNA was extracted from placental tissue using TRIzol ${ }^{\circledR}$ reagent (Invitrogen; Thermo Fisher Scientific, Inc.) and $5 \mu \mathrm{g}$ RNA was reverse transcribed into cDNA using an All-in-one ${ }^{\mathrm{TM}}$ First-Strand cDNA Synthesis kit (Guangzhou Fansi Biotechnology Co., Ltd) according to the manufacturers' protocol. Subsequently, qPCR was performed using a SYBR Green Realtime PCR Mix kit (Mei5bio; Beijing Jumei Biotechnology Co., Ltd.). Gene accession numbers were 51099 for CGI-58 (www.ncbi.nlm.nih.gov/gene/51099) and 4023 for LPL (www.ncbi.nlm.nih.gov/gene/4023). The sequences of the primers used were as follows: CGI-58, forward 5'-ATC AAGGGTTAATCATCTCA-3', reverse 5'-CTGGAATTG GTCTGTCTT-3'; LPL, forward 5'-CATAGCCTATAATTG GTTAG-3, reverse 5'-GTGTAGATGAGTCTGATT-3'; and $\beta$-actin, forward 5'-ATATGAGATGCGTTGTTA-3' and reverse 5'-AAGTATTAAGGCGAAGAT-3'. $\beta$-actin was used as the internal reference. The thermocycling conditions were as follows: Initial denaturation at $95^{\circ} \mathrm{C}$ for $10 \mathrm{~min}$; followed by 40 cycles of $95^{\circ} \mathrm{C}$ for $15 \mathrm{sec}, 60^{\circ} \mathrm{C}$ for $20 \mathrm{sec}, 72^{\circ} \mathrm{C}$ for $30 \mathrm{sec}$ and $60^{\circ} \mathrm{C}$ for $1 \mathrm{~min}$. The relative expression levels of CGI-58 and LPL were normalized to the internal reference control $\beta$-actin and quantified using the $2^{-\Delta \Delta \mathrm{Cq}}$ method $(\Delta \mathrm{Cq}=\mathrm{Cq}$ value of CGI-58 or LPL-Cq value of $\beta$-actin; $\Delta \Delta \mathrm{Cq}=\mathrm{Cq}$ value of preeclampsia-Cq value of control) (28).

Immunohistochemistry analysis. Paraffin-embedded placental tissue samples with thickness of $\sim 3 \mathrm{~mm}$ were successively dewaxed and rehydrated using xylene, ethanol (100, 95, 85 and $75 \%$ ) and tap water. Sections were immersed in $0.01 \mathrm{~mol} / 1$ sodium citrate buffer above $100^{\circ} \mathrm{C}$ for antigen retrieval, and peroxide activity was quenched using $3 \% \mathrm{H}_{2} \mathrm{O}_{2}$ at room temperature for $15 \mathrm{~min}$. Subsequently, sections were incubated separately with either rabbit anti-human CGI-58 antibody
(1:100; cat. no. DF12065; Affinity Biosciences) or rabbit anti-human LPL antibody (1:100; cat. no. A9228; ABclonal) at $4^{\circ} \mathrm{C}$ for $12 \mathrm{~h}$. Then, sections were incubated with goat anti-rabbit IgG horseradish peroxidase-conjugated secondary antibody (1:100; cat. no. ab97051; Abcam.) for $30 \mathrm{~min}$ at $37^{\circ} \mathrm{C}$. Samples were washed with PBS and stained using a DAB Staining kit (Sigma-Aldrich; Merck KGaA) according to the manufacturers' protocol. The samples were counterstained with hematoxylin, rinsed, dehydrated (70, 80, 95 and $100 \%$ ethanol, analytical reagent grade) and sealed with neutral resin sequentially. The immunohistochemical staining was imaged using a Micro Publisher 5.0 microscope (Roper Industries; magnification, $\mathrm{x} 400$ ) and staining was analyzed using Image-Pro-Plus 6.0 software (Media Cybernetics, Inc.).

The CGI-58 and LPL staining were classified as positive or negative, depending on the presence or absence of brownish yellow granules in the cell membrane and cytoplasm, respectively. A total of 10 randomly selected fields of view were selected to count the total number of cells and the number of positive cells. The positive cell rate $(\%)$ was calculated using the following formula: (Number of positive cells/total number of cells) x100. If the positive cell rate was $>10 \%$, the sample was considered as positive (+), otherwise, it was considered as negative.

Western blotting. Total proteins from placental tissue were extracted using RIPA protein lysate (Beyotime Institute of Biotechnology) on ice and quantified using a BCA Protein assay kit (Beyotime Institute of Biotechnology) according to the manufacturers' protocols. Proteins $(15 \mu \mathrm{g})$ were denatured and separated by $12 \%$ SDS-PAGE and transferred onto a PVDF membrane at $100 \mathrm{~V}$ for $2 \mathrm{~h}$. Once nonspecific binding was blocked using $5 \%$ skimmed milk at $4^{\circ} \mathrm{C}$ overnight, membranes were incubated with the rabbit anti-human primary antibodies against CGI-58 (1:1,000; cat. no. DF12065; Affinity Biosciences), LPL (1:1,000; cat. no. A9228; ABclonal) and $\beta$-actin (1:3,000; cat. no. ab8226; Abcam) overnight at $4^{\circ} \mathrm{C}$. Membranes were then incubated with goat anti-rabbit IgG secondary antibody (1:3,000; cat. no. ab97051; Abcam) at room temperature for $2 \mathrm{~h}$. Eventually, antibody binding was detected using enhanced chemiluminescence substrate (National Diagnostic) and visualized using autoradiography. Relative expression levels of CGI-58 and LPL were normalized to endogenous control $\beta$-actin using Image Lab v.5.0 software (Bio-Rad Laboratories, Inc.).

Statistical analysis. SPSS version 17.0 (SPSS, Inc.) was used for statistical analysis. Data are presented as the means \pm standard deviation and compared using a Student's t-test. A $\chi^{2}$ test was used to analyze the classified count data. Pearson's linear correlation analysis was used to analyze the relationship between two variables. A multivariate logistic regression model was used for multivariate analysis. $\alpha=0.05$ was used as the test level (bilateral) and $\mathrm{P}<0.05$ was considered to indicate a statistically significant difference.

\section{Results}

Analysis of clinical data. As presented in Table I, there were no significant differences in age, gestational week, pregnancy time, production time and neonatal weight between the preeclampsia 
Table I. Clinical data analysis of the preeclampsia and control groups.

\begin{tabular}{lccc}
\hline Variable & Preeclampsia $(\mathrm{n}=37)$ & Control $(\mathrm{n}=40)$ & P-value \\
\hline Age, years & $29.85 \pm 4.78$ & $28.69 \pm 3.60$ & 0.21 \\
Gestational week, weeks & $37.8 \pm 0.63$ & $39.1 \pm 1.25$ & 0.08 \\
Number of pregnancies, $\mathrm{n}$ & $1.85 \pm 0.87$ & $1.90 \pm 1.30$ & 0.81 \\
Number of births, $\mathrm{n}$ & $1.41 \pm 0.54$ & $1.36 \pm 0.49$ & 0.61 \\
Uterine height, cm & $34.52 \pm 3.82$ & $32.88 \pm 2.30$ & $0.02^{\mathrm{a}}$ \\
Abdominal circumference, $\mathrm{cm}$ & $106.61 \pm 9.58$ & $97.95 \pm 15.05$ & $0.00^{\mathrm{a}}$ \\
Neonatal weight, g & $3,105.20 \pm 583.33$ & $3,295.33 \pm 385.57$ & 0.08 \\
BMI before pregnancy & $24.46 \pm 5.06$ & $22.27 \pm 3.15$ & $0.02^{\mathrm{a}}$ \\
Gestational weight gain, $\mathrm{kg}$ & $18.48 \pm 5.96$ & $14.01 \pm 4.74$ & $0.00^{\mathrm{a}}$ \\
\hline
\end{tabular}

${ }^{\mathrm{a}} \mathrm{P}<0.05$. BMI, body mass index.

Table II. Comparison of blood lipid levels between preeclampsia and control groups.

\begin{tabular}{lccc}
\hline Blood lipids & $\begin{array}{c}\text { Preeclampsia } \\
(\mathrm{n}=37)\end{array}$ & $\begin{array}{c}\text { Control } \\
(\mathrm{n}=40)\end{array}$ & P-value \\
\hline $\mathrm{TC}, \mathrm{mmol} / \mathrm{l}$ & $6.19 \pm 1.23$ & $5.93 \pm 1.09$ & 0.30 \\
$\mathrm{TG}, \mathrm{mmol} / \mathrm{l}$ & $3.57 \pm 1.09$ & $2.83 \pm 1.08$ & $0.00^{\mathrm{a}}$ \\
$\mathrm{HDL}-\mathrm{C}, \mathrm{mmol} / \mathrm{l}$ & $1.76 \pm 0.50$ & $2.02 \pm 0.38$ & $0.01^{\mathrm{a}}$ \\
$\mathrm{LDL}-\mathrm{C}, \mathrm{mmol} / \mathrm{l}$ & $3.57 \pm 0.78$ & $3.21 \pm 0.78$ & $0.03^{\mathrm{a}}$ \\
$\mathrm{Lp}(\mathrm{a}), \mathrm{mg} / \mathrm{l}$ & $205.17 \pm 92.05$ & $135.74 \pm 83.48$ & 0.07 \\
ApoA, g/l & $2.01 \pm 0.34$ & $2.18 \pm 0.26$ & $0.01^{\mathrm{a}}$ \\
ApoB, g/l & $1.28 \pm 0.27$ & $1.14 \pm 0.29$ & $0.02^{\mathrm{a}}$ \\
AI & $3.88 \pm 0.97$ & $2.95 \pm 1.14$ & $0.00^{\mathrm{a}}$ \\
\hline
\end{tabular}

${ }^{\mathrm{a}} \mathrm{P}<0.05$. AI, atherosclerotic index; ApoA, apolipoprotein A; ApoB, apolipoprotein B; HDL-C, high density lipoprotein-cholesterol; LDL-C, low density lipoprotein-cholesterol; Lp(a), lipoprotein small a; TC, total cholesterol; TG, triglycerides.

and control groups (all $\mathrm{P}>0.05$ ). However, the uterine height, abdominal circumference, BMI before pregnancy and gestational weight gain in the preeclampsia group were significantly higher compared with the control group $(\mathrm{P}<0.05)$.

A seen in Table II, there was no significant difference in TC and $\mathrm{Lp}$ (a) levels between the preeclampsia and control groups $(\mathrm{P}>0.05)$. The TG, LDL-C and ApoB levels in the preeclampsia group were significantly increased compared with those in the control group (all $\mathrm{P}<0.05$ ). HDL-C and ApoA levels in the preeclampsia group were significantly decreased compared with the control group $(\mathrm{P}<0.05)$. As an index to predict atherosclerosis, the atherosclerotic index (AI) can directly reflect the degree of lipid metabolism disorder, and was calculated as follows: $\mathrm{AI}=(\mathrm{TC}-\mathrm{HDL}-\mathrm{C}) / \mathrm{HDL}-\mathrm{C}$. The AI value of the preeclampsia group was significantly higher than that of the control group $(\mathrm{P}<0.05)$.

CGI-58 and LPL levels. The expression levels of CGI-58 and LPL in placental tissues were detected using RT-qPCR. As presented in Figs. 1 and 2, the relative expression of both CGI-58

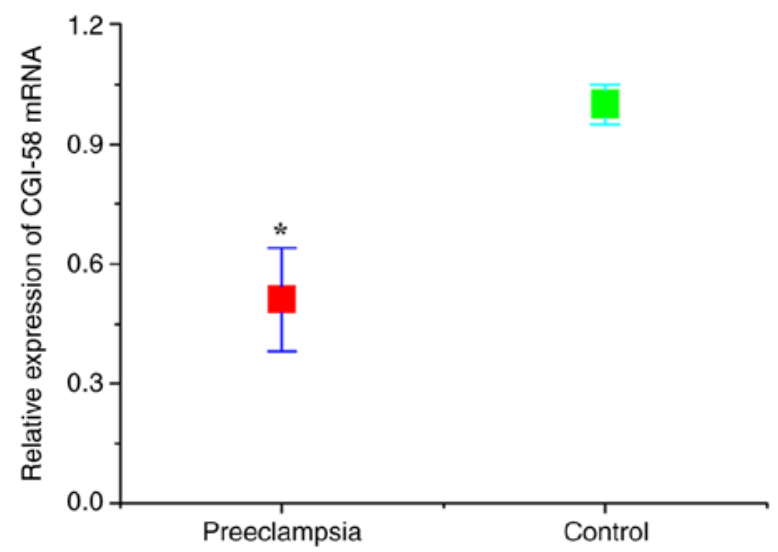

Figure 1. Relative expression of CGI-58 mRNA in the placentas of the preeclampsia $(n=37)$ and control (normal pregnant, $n=40)$ groups evaluated by reverse transcription quantitative PCR. " $\mathrm{P}<0.05$ vs. control. CGI-58, comparative gene identification-58.

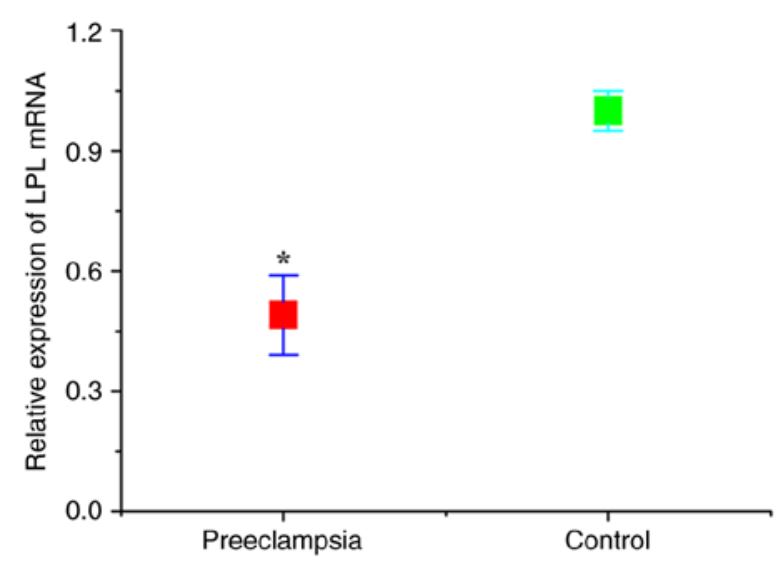

Figure 2. Relative expression of LPL mRNA in the placentas of the preeclampsia $(n=37)$ and control (normal pregnant, $n=40)$ groups evaluated by reverse transcription quantitative PCR. ${ }^{*} \mathrm{P}<0.05$ vs. control. LPL, lipoprotein lipase.

and LPL mRNA in placental tissues of the preeclampsia group was significantly lower than that of control (normal pregnant) group (both $\mathrm{P}<0.05$ ). The expression levels of CGI-58 and LPL 

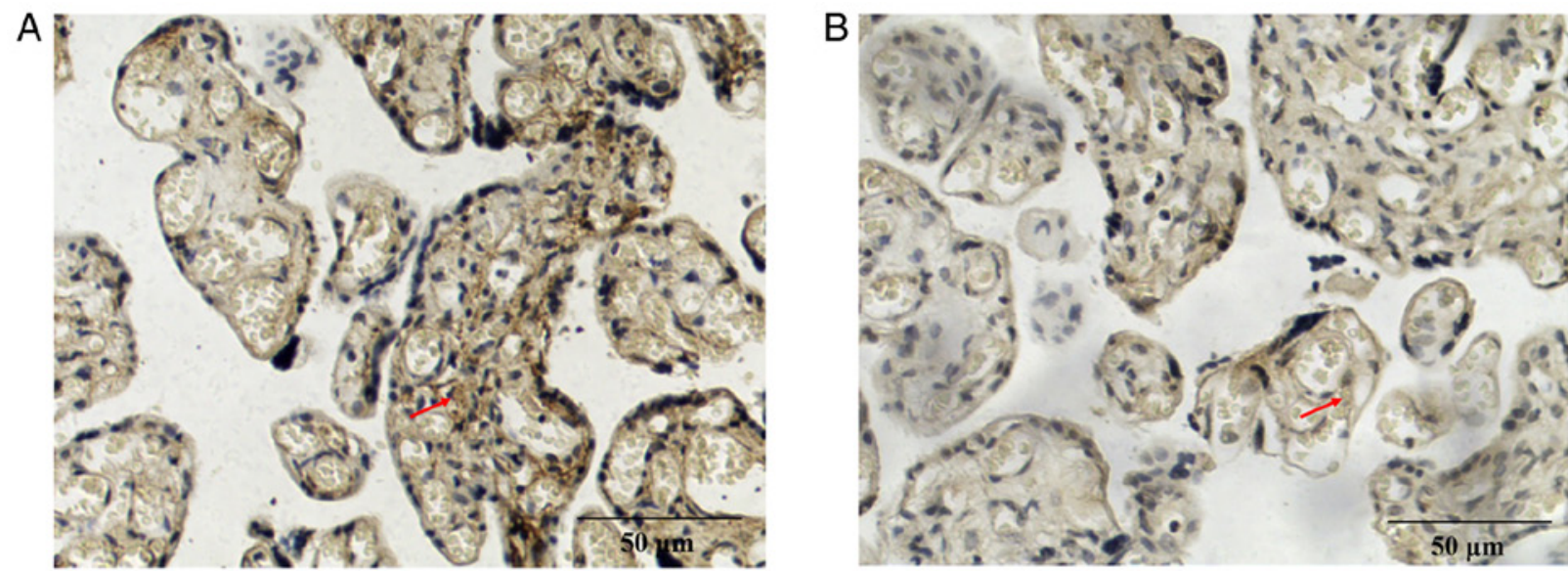

Figure 3. Immunohistochemical examination of CGI-58 in the (A) preeclampsia and (B) control (normal pregnant) groups. Samples were considered CGI-58-positive when brown-yellow granules were detected in the cytoplasm and cell membrane (red row). Magnification, $\mathrm{x} 400$. Scale bar, 50- $\mu \mathrm{m}$. CGI-58, comparative gene identification-58.
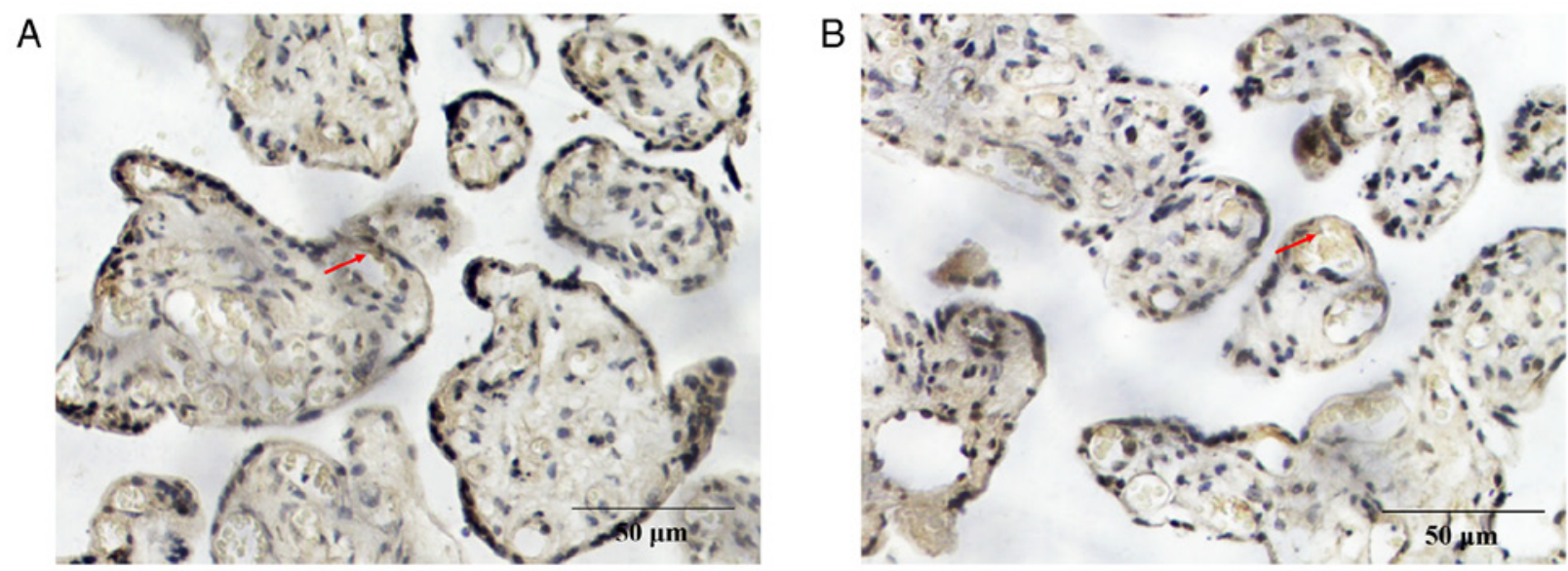

Figure 4. Immunohistochemical examination for LPL in the (A) preeclampsia and (B) control (normal pregnant) groups. Samples were considered LPL-positive when brown-yellow granules were detected in the cytoplasm and cell membrane (red row). Magnification, x400. Scale bar, 50- $\mu \mathrm{m}$. LPL, lipoprotein lipase.

in placental tissue were further assessed using immunohistochemistry. The positive expression of CGI-58 (Fig. 3) and LPL (Fig. 4) was characterized by brown and yellow granules in the cell membrane and cytoplasm, and their expression was weakly positive in the preeclampsia group (Figs. 3A and 4A) and positive in the control group (Figs. 3B and 4B). The positive rates of CGI-58 and LPL were 40.54 and $29.73 \%$, respectively, in the preeclampsia group, which were both significantly lower compared with the control group (72.50 and 67.50\%, respectively; $\mathrm{P}<0.05$; Table III). Semi quantitative western blot method was also used to test the expression of CGI-58 and LPL (Fig. 5). The expression of both CGI-58 and LPL in the placental tissue of the preeclampsia group was significantly lower than in the control group $(\mathrm{P}<0.05$; Figs. 6 and 7). The results from RT-qPCR, immunohistochemistry and western blotting were therefore consistent.

Association analysis. As presented in Table IV, the mean protein expression levels of CGI-58 (0.75) and LPL (0.59) in placental tissue were selected as the threshold between high and low expression. A $\chi^{2}$ test revealed that the expression levels of CGI-58 and LPL were associated with preeclampsia. The risk of preeclampsia in pregnant women with high expression levels of CGI-58 and LPL was only 0.19 and 0.22 in pregnant women with low expression levels of CGI-58 and LPL, respectively. These results demonstrated that low expression of CGI-58 and LPL are risk factors for preeclampsia in pregnant women.

To investigate the risk factors of preeclampsia, the indicators that differed significantly between the preeclampsia and control groups, including uterine height, abdominal circumference, BMI before pregnancy, gestational weight gain, TG, HDL-C, LDL-C, ApoA, ApoB, AI, CGI-58 and LPL levels, were selected as independent variables, and their corresponding means $(33.73,102.47,23.41,16.34,3.22$, $1.88,3.40,2.08,1.21,3.42,0.75$ and 0.59 , respectively) were used as the threshold between high and low expression. As presented in Table $\mathrm{V}$, multivariate logistic regression analysis demonstrated that gestational weight gain, TG, AI, CGI-58 and LPL levels were significant different (all $\mathrm{P}<0.05)$, and the odds ratios were $7.61,4.60,5.98,1.19$ and 1.23 , respectively. Their corresponding $95 \%$ confidence intervals were $2.22-26.04$, $1.36-15.54,1.87-19.09,1.02-1.39$ and 1.05-2.20, respectively.

Pearson's linear correlation analysis was used to study the association between the expression of CGI-58 and LPL in the 
Table III. Immunohistochemical examination for CGI-58 and LPL in placenta of preeclampsia and control groups.

\begin{tabular}{llccccc}
\hline \multirow{2}{*}{ Protein } & & & \multicolumn{2}{c}{ Number of specimens } & & \\
& Groups & Number & Negative expression & Positive expression & Positive rate (\%) & P-value \\
\hline CGI-58 & Preeclampsia & 37 & 22 & 15 & 40.54 & $0.01^{\mathrm{a}}$ \\
& Control & 40 & 11 & 29 & 72.50 & \\
\multirow{2}{*}{ LPL } & Preeclampsia & 37 & 26 & 11 & 29.73 & $0.00^{\mathrm{a}}$ \\
& Control & 40 & 13 & 27 & 67.50 & \\
\hline
\end{tabular}

${ }^{\mathrm{a}} \mathrm{P}<0.05$. CGI-58, comparative gene identification-58; LPL, lipoprotein lipase.

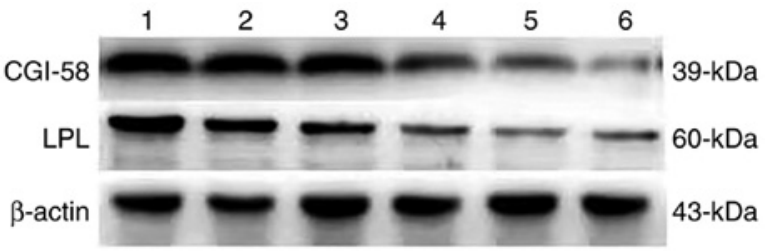

Figure 5. Representative expression of CGI-58 and LPL in control (normal pregnant, column 1,2,3) and preeclampsia (column 4, 5, 6) groups evaluated by western blotting. CGI-58, comparative gene identification-58; LPL, lipoprotein lipase.

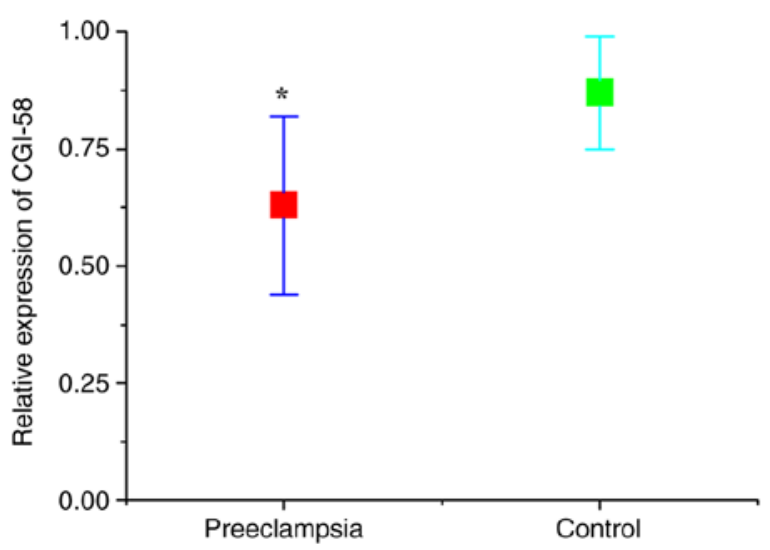

Figure 6. Relative CGI-58 protein expression calculated from the results from western blotting (Fig. 5) in the placentas of preeclampsia $(n=37)$ and control (normal pregnant, $\mathrm{n}=40$ ) groups. ${ }^{*} \mathrm{P}<0.05$ vs. control. CGI-58, comparative gene identification-58.

placenta and the blood lipid levels. As presented in Table VI, CGI-58 was positively correlated with HDL-C $(r=0.63$; $\mathrm{P}<0.01)$, negatively correlated with $\mathrm{TG}$ and ApoB $(\mathrm{r}=0.84$; $\mathrm{P}<0.01 ;$ and $\mathrm{r}=0.51 ; \mathrm{P}<0.05$, respectively), and not correlated with TC, LDL-C, Lp(a), ApoA and AI (P>0.05). CGI-58 was positively correlated with $\mathrm{HDL}-\mathrm{C}(\mathrm{r}=0.52 ; \mathrm{P}<0.01)$ and negatively correlated with TG and AI $(r=0.66 ; \mathrm{P}<0.01$; and $\mathrm{r}=0.47 ; \mathrm{P}<0.05$, respectively). In addition, CGI-58 was positively correlated with LPL $(r=0.60 ; \mathrm{P}<0.05)$.

\section{Discussion}

Preeclampsia is an idiopathic pregnancy disease which seriously endangers the life of both the mother and the infant.

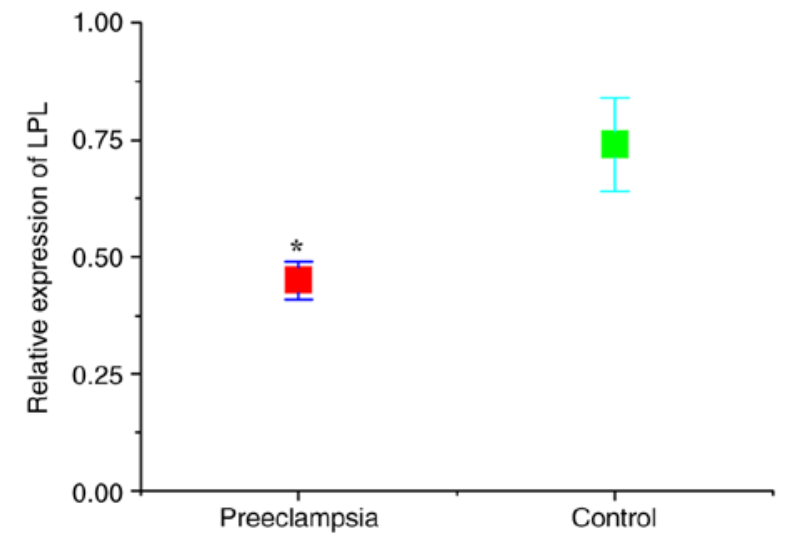

Figure 7. Relative LPL protein expression calculated from the results from western blotting (Fig. 5) in the placentas of preeclampsia and control (normal pregnant) groups. ${ }^{\mathrm{P}}<0.05$ vs. control. LPL, lipoprotein lipase.

Although the etiology of preeclampsia has not been fully elucidated, it is established that abnormal lipid metabolism during pregnancy serves an important role in its pathogenesis $(29,30)$. Disorders of lipid metabolism and abnormal transformation of lipoproteins can cause atherosclerosis, leading to the accumulation of lipid peroxides, promoting oxidative stress and aggravating damage to vascular endothelial function (31). Acute atherosclerotic changes are observed in $20-40 \%$ of the placental tissues of patients with preeclampsia, in which the vascular resistance of the spiral artery increases and microthrombosis leads to placental villi infarction and therefore, a decrease in placental perfusion (32). TG in maternal blood is converted into fatty acids via the placenta, which is an important source of energy for the fetus. CGI-58 and LPL are involved in the hydrolysis of TG, and their abnormal expression can result in lipid transport disorders in the placenta and affect the regulation of placental metabolism and energy supply (13). In the present study, the expression of CGI-58 and LPL in the placenta were therefore detected to examine the relationship between CGI-58 and LPL expression and the pathogenesis of preeclampsia.

To meet the needs of fetal growth and development during pregnancy, the maternal blood lipid levels increase gradually with time (11). Unlike the changes in blood lipids during normal pregnancy, the higher blood lipids level in preeclampsia pregnant women lead to atherosclerotic changes, which can cause oxidative stress due to high production of lipid peroxides and 
Table IV. Relationship between preeclampsia and the expression of CGI-58 and LPL in placenta.

\begin{tabular}{|c|c|c|c|c|c|c|c|}
\hline Factors & $\mathrm{n}$ & High level & Low level & $\chi^{2}$ & P-value & OR & $95 \% \mathrm{CI}$ \\
\hline CGI-58 & & $\geq 0.75$ & $<0.75$ & & & & \\
\hline Preeclampsia & 37 & 10 & 27 & 11.13 & $0.00^{\mathrm{b}}$ & 0.20 & $0.15-0.65$ \\
\hline Control & 40 & 26 & 14 & & & & \\
\hline $\mathrm{LPL}^{\mathrm{a}}$ & & $\geq 0.59$ & $<0.59$ & & & & \\
\hline Preeclampsia & 37 & 13 & 24 & 5.76 & $0.02^{\mathrm{b}}$ & 0.33 & $0.17-0.75$ \\
\hline Control & 40 & 25 & 15 & & & & \\
\hline
\end{tabular}

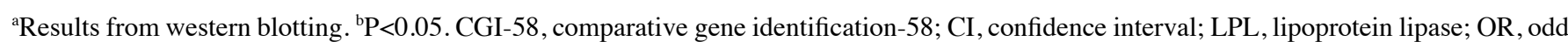
ratio.

Table V. Multivariate logistic regression analysis of factors affecting the development of preeclampsia.

\begin{tabular}{|c|c|c|c|c|c|c|}
\hline Factors & $\beta$ & SE & Wald & P-value & OR & $95 \% \mathrm{CI}$ \\
\hline Gestational weight gain, $\mathrm{kg}$ & 2.03 & 0.63 & 10.45 & $0.00^{\mathrm{a}}$ & 7.61 & $2.22-26.04$ \\
\hline $\mathrm{TG}, \mathrm{mmol} / \mathrm{l}$ & 1.53 & 0.62 & 6.02 & $0.01^{\mathrm{a}}$ & 4.60 & $1.36-15.54$ \\
\hline AI & 1.79 & 0.59 & 9.11 & $0.00^{\mathrm{a}}$ & 5.98 & $1.87-19.09$ \\
\hline CGI-58 & 0.17 & 0.08 & 4.76 & $0.03^{\mathrm{a}}$ & 1.19 & $1.02-1.39$ \\
\hline LPL & 0.21 & 0.09 & 5.62 & $0.04^{\mathrm{a}}$ & 1.23 & $1.05-2.20$ \\
\hline
\end{tabular}

${ }^{\text {aP }}<0.05$. AI, atherosclerotic index; CGI-58, comparative gene identification-58; CI, confidence interval; LPL, lipoprotein lipase; OR, odd ratio; TG, triglycerides.

Table VI. Correlation analysis between CGI-58 and LPL expression in placenta and maternal blood lipid levels.

\begin{tabular}{lccccc}
\hline & \multicolumn{3}{c}{ CGI-58 } & & \multicolumn{2}{c}{ LPL } \\
\cline { 2 - 3 } \cline { 5 - 6 } Factor & $\mathrm{r}$ & P-value & & $\mathrm{r}$ & P-value \\
\hline TC & -0.278 & 0.249 & -0.187 & 0.469 \\
TG & -0.840 & 0.000 & & -0.659 & $0.000^{\mathrm{a}}$ \\
HDL-C & 0.625 & 0.003 & & 0.524 & $0.009^{\mathrm{a}}$ \\
LDL-C & -0.373 & 0.174 & -0.372 & 0.095 \\
Lp(a) & -0.312 & 0.210 & -0.234 & 0.249 \\
ApoA & 0.393 & 0.097 & & 0.313 & 0.186 \\
ApoB & -0.514 & 0.026 & -0.428 & 0.074 \\
AI & -0.486 & 0.063 & -0.466 & 0.038
\end{tabular}

${ }^{\mathrm{a}} \mathrm{P}<0.05$. AI, atherosclerotic index; ApoA, apolipoprotein A; ApoB, apolipoprotein B; CGI-58, comparative gene identification-58; HDL-C, high density lipoprotein-cholesterol; LDL-C, low density lipoprotein-cholesterol; Lp(a), lipoprotein small a; LPL, lipoprotein lipase; TC, total cholesterol; TG, triglycerides.

the subsequent release of reactive oxygen species, resulting in decreased vasodilation and increased endothelial damage (33). Wojcik-Baszko et al (12) reported that the levels of TC and TG in preeclampsia pregnant women are $>205$ and $>133 \mathrm{mg} / \mathrm{dl}$, which are higher than those in normal pregnant women by 3.6x and 4.15x, respectively. LDL-C and HDL-C are also increased by $10.4 \%$ and decreased by $7 \%$, respectively. In the present study, the serum levels of TG, LDL-C, ApoB and AI in the preeclampsia group were significantly higher than those in the control group, whereas the serum levels of HDL-C and ApoA in the preeclampsia group were lower than those in the control group, which was consistent with a previous study (18).

Being overweight or obese are the most important risk factors for preeclampsia with attributable risk percentages of 64.9 and $64.4 \%$, respectively (34). However, the relationship between obesity and the onset of preeclampsia has not been fully elucidated. Obese pregnant women may develop preeclampsia through a lipid metabolism disorder (35). In the present study, the BMI before pregnancy and the gestational weight gain of preeclampsia patients were significantly higher than those of normal pregnant women. The weight gain during pregnancy was an independent risk factor of preeclampsia. With an increase in gestational weight gain, the risk of preeclampsia increases. The unbalanced diet and excessive intake of fat and other nutrients during pregnancy lead to excessive weight gain, which may be important factors causing lipid metabolism disorder. Weight control and proper physical exercise during pregnancy can increase insulin sensitivity, relieve sympathetic nervous tension and reduce serum TG concentration and blood glucose levels (36). These measures may help obese people reducing their risk of preeclampsia. The detection of blood lipid levels can help identifying high-risk pregnant women earlier, allowing a better tracking and targeted preventative interventions and treatments, in order to reduce maternal mortality caused by preeclampsia. 
Fatty acids are the key substances for fetal brain development and adipose tissue formation. Maternal TG is the most important source of fetal fatty acids, and $20-50 \%$ of fetal fatty acids come from the maternal circulation (7). TG is present in adipocytes in the form of lipid droplets, which cannot pass through the cell membrane directly. TG must be hydrolyzed by lipase to be transported in vivo and deposited in adipose tissue or directly used for energy. LPL participates in the hydrolysis of TG and promotes the transport of lipids to embryos. CGI-58 can increase the catalytic activity of LPL by 20x (13). Disorders of placental lipid transport can lead to lipid accumulation and increased lipid peroxide formation, resulting in endothelial cell injury (37). In the present study, the mRNA and protein expression of CGI-58 and LPL in placenta was decreased, and their expression levels were positively correlated, which may have led to the accumulation of lipid hydrolysates and oxidative stress reactions via TG metabolism and lipid transport.

Dyslipidemia and abnormal expression of lipoproteins may be closely associated with the pathogenesis of preeclampsia. Disorders of placental lipid transport can lead to lipid accumulation and the production of a large number of peroxides, which can cause endothelial cell damage (16). In addition, inflammatory factors released by the placenta can cause systemic vascular endothelial dysfunction (12). In pregnant women with preeclampsia, glomerular endothelial hyperplasia and alterations to the umbilical vein and placental uterine vascular endothelial cells are observed (38). The levels of markers of endothelial cell activation in serum, including adhesion molecules, cytokines, procoagulant factors and antiangiogenic factors are increased (16). Oxidative stress at the placental interface can lead to disordered placental spiral artery remodeling, resulting in placental dysplasia, insufficient trophoblast cell infiltration and preeclampsia (31). CGI-58 and LPL are involved in the first step of triglyceride hydrolysis and serve an important role in lipid transport in the placenta (13). In the present study, CGI-58 expression was positively correlated with maternal serum HDL-C levels and negatively correlated with TG and ApoB levels. Placental LPL expression was positively correlated with HDL-C and negatively correlated with TG level and AI. These results suggested that the expression of CGI-58 and LPL in the placenta may be associated with disorders of maternal lipid metabolism. As placental detection can only be performed following delivery and since the expression of CGI-58 and LPL in the placenta is associated with maternal blood lipid levels, blood lipid levels during pregnancy may be used to assist the diagnosis of preeclampsia. In addition, maternal serum CGI-58 and LPL expression levels may also be associated with preeclampsia or maternal blood lipid levels, and both these hypotheses will be assessed. In future studies, the expression levels of maternal CGI-58 and LPL will be assessed to determine the relationship between lipid metabolism and the expression levels of CGI-58 and LPL and to further clarify the mechanism by which upregulated expression of CGI-58 and LPL can increase the risk of preeclampsia.

In conclusion, compared with normal pregnant women, TG, LDL-C, ApoB levels and AI in the serum of preeclampsia pregnant women were significantly increased, whereas HDL-C and ApoA levels were significantly decreased, suggesting that patients with preeclampsia exhibited dyslipidemia. CGI-58 and LPL expression in placental tissue of preeclampsia pregnant women was decreased, and the expression levels of CGI-58 and LPL were positively correlated. Therefore, the risk of preeclampsia was increased when the expression levels of CGI-58 or LPL were decreased. It is hypothesized that CGI-58 and LPL in the placenta may affect lipid metabolism in the serum and placenta, and that CGI-58 and LPL may be considered as important indicators for the diagnosis of preeclampsia. CGI-58 and LPL could therefore serve an important role in the pathogenesis of preeclampsia, which may be related to the accumulation of lipid peroxides in the placenta of preeclampsia women, leading to increased systemic oxidative stress.

\section{Acknowledgements}

Not applicable.

\section{Funding}

No funding was received.

\section{Availability of data and materials}

The datasets used and/or analyzed during the current study are available from the corresponding author on reasonable request.

\section{Authors' contributions}

JD and YC designed the study. JD, MW and JG performed the experiments. JD and JL analyzed the data. JD and YC wrote the manuscript. JD and YC confirmed the authenticity of all the raw data. All authors read and approved the final manuscript.

\section{Ethics approval and consent to participate}

The present study was approved by the Ethics Committee of the North China University of Science and Technology Affiliated Hospital (approval no. 20180718A). Informed consent was obtained from all patients.

\section{Patient consent for publication}

Not applicable.

\section{Competing interests}

The authors declare that they have no competing interests.

\section{References}

1. Rana S, Lemoine E, Granger JP and Karumanchi SA: Preeclampsia: Pathophysiology, challenges, and perspectives. Circ Res 124: 1094-1112, 2019.

2. De Kat AC, Hirst J, Woodward M, Kennedy S and Peters SA: Prediction models for preeclampsia: A systematic review. Pregnancy Hypertens 16: 48-66, 2019.

3. Staff AC: The two-stage placental model of preeclampsia: An update. J Reprod Immunol 134-135: 1-10, 2019.

4. Perucci LO, Corrêa MD, Dusse LM, Gomes KB and Sousa LP: Resolution of inflammation pathways in preeclampsia-a narrative review. Immunol Res 65: 774-789, 2017. 
5. Sheridan MA, Yang Y, Jain A, Lyons AS, Yang P, Brahmasani SR, Dai A, Tian Y, Ellersieck MR, Tuteja G, et al: Early onset preeclampsia in a model for human placental trophoblast. Proc Natl Acad Sci USA 116: 4336-4345, 2019.

6. Wang T, Shi XZ and Wu WH: Crosstalk analysis of dysregulated pathways in preeclampsia. Exp Ther Med 17: 2298-2304, 2019.

7. Geldenhuys J, Rossouw TM, Lombaard HA, Ehlers MM and Kock MM: Disruption in the regulation of immune responses in the placental subtype of preeclampsia. Front Immunol 9: 1659, 2018.

8. Travaglino A, Raffone A, Saccone G, Migliorini S, Maruotti GM, Esposito G, Mollo A, Martinelli P, Zullo F and D'Armiento M: Placental morphology, apoptosis, angiogenesis and epithelial mechanisms in early-onset preeclampsia. Eur J Obstet Gynecol Reprod Biol 234: 200-2006, 2019.

9. Valencia-Ortega J, Zárate A, Saucedo R, Hernández-Valencia M, Cruz JG and Puello E: Placental proinflammatory state and maternal endothelial dysfunction in preeclampsia. Gynecol Obstet Invest 84: 12-19, 2019.

10. Yang H, He B, Yallampalli C and Gao H: Fetal macrosomia in a hispanic/Latinx predominant cohort and altered expressions of genes related to placental lipid transport and metabolism. Int J Obes (Lond) 44: 1743-1752, 2020.

11. Lewis RM, Wadsack C and Desoye G: Placental fatty acid transfer. Curr Opin Clin Nutr Metab Care 21: 78-82, 2018.

12. Wojcik-Baszko D, Charkiewicz K and Laudanski P: Role of dyslipidemia in preeclampsia-A review of lipidomic analysis of blood, placenta, syncytiotrophoblast microvesicles and umbilical cord artery from women with preeclampsia. Prostaglandins Other Lipid Mediat 139: 19-23, 2018.

13. Kulminskaya N and Oberer M: Protein-protein interactions regulate the activity of adipose triglyceride lipase in intracellular lipolysis. Biochimie 169: 62-68, 2020

14. Benschop L, Bergen NE, Schalekamp-Timmermans S, Jaddoe VW, Mulder MT, Steegers EAP and van Lennep JE: Maternal lipid profile 6 years after a gestational hypertensive disorder. J Clin Lipidol 12: 428-436, 2018.

15. Onda K, Tong S, Beard S, Binder N, Muto M, Senadheera SN, Parry L, Dilworth M, Renshall L, Brownfoot F, et al: Proton pump inhibitors decrease soluble fms-like tyrosine kinase-1 and soluble endoglin secretion, decrease hypertension, and rescue endothelial dysfunction. Hypertension 69: 457-468, 2017.

16. Townsend R, Khalil A, Premakumar Y, Allotey J, Snell KIE Chan C, Chappell LC, Hooper R, Green M, Mol BW, et al: Prediction of pre-eclampsia: Review of reviews. Ultrasound Obstet Gynecol 54: 16-27, 2019.

17. Lorentzen B, Drevon CA, Endresen MJ and Henriksen T: Fatty acid pattern of esterified and free fatty acids in sera of women with normal and pre-eclamptic pregnancy. Br J Obstet Gynaecol 102: 530-537, 1995.

18. Olivecrona G: Role of lipoprotein lipase in lipid metabolism Curr Opin Lipidol 27: 233-241, 2016.

19. Colvin BN, Longtine MS, Chen B, Costa ML and Nelson DM: Oleate attenuates palmitate-induced endoplasmic reticulum stress and apoptosis in placental trophoblasts. Reproduction 153 : 369-380, 2017.

20. Li B, Fang J, He T, Yin S, Yang M, Cui H, Ma X, Deng J, Ren Z, $\mathrm{Hu}$ Y, et al: Resistin up-regulates LPL expression through the PPAR $\gamma$-dependent PI3K/AKT signaling pathway impacting lipid accumulation in RAW264.7 macrophages. Cytokine 119: 168-174, 2019.

21. Oberer M, Boeszoermenyi A, Nagy HM and Zechner R: Recent insights into the structure and function of comparative gene identification-58. Curr Opin Lipidol 22: 149-158, 2011.

22. Zhang J, Xu D, Nie J, Han R, Zhai Y and Shi Y: Comparative gene identification-58 (CGI-58) promotes autophagy as a putative lysophosphatidylglycerol acyltransferase. J Biol Chem 289: 33044-33053, 2014.

23. Goeritzer M, Schlager S, Radovic B, Madreiter CT, Rainer S, Thomas G, Lord CC, Sacks J, Brown AL, Vujic N, et al: Deletion of CGI-58 or adipose triglyceride lipase differently affects macrophage function and atherosclerosis. J Lipid Res 55: $2562-2575,2014$
24. Miao H, Ou J, Ma Y, Guo F, Yang Z, Wiggins M, Liu C, Song W, Han X, Wang M, et al: Macrophage CGI-58 deficiency activates ROS-inflammasome pathway to promote insulin resistance in mice. Cell Rep 7: 223-235, 2014.

25. Zhang S, Liu G, Xu C, Liu L, Zhang Q, Xu Q, Jia H, Li X and Li X: Perilipin 1 mediates lipid metabolism homeostasis and inhibits inflammatory cytokine synthesis in bovine adipocytes. Front Immunol 9: 467, 2018.

26. Contini C, Jansen M, König B, Markfeld-Erol F, Kunze M, Zschiedrich S, Massing U, Merfort I, Prömpeler H, Pecks U, et al: Lipoprotein turnover and possible remnant accumulation in preeclampsia: Insights from the freiburg preeclampsia H.E.L.P.-apheresis study. Lipids Health Dis 17: 49, 2018

27. Chiarello DI, Abad C, Rojas D, Toledo F, Vázquez CM, Mate A, Sobrevia L and Marín R: Oxidative stress: Normal pregnancy versus preeclampsia. Biochim Biophys Acta Mol Basis Dis 1866: $165354,2020$.

28. Livak KJ and Schmittgen TD: Analysis of relative gene expression data using real-time quantitative PCR and the 2(-Delta Delta C(T)) method. Methods 25: 402-408, 2001

29. Cao W, Wang X, Chen T, Xu W, Feng F, Zhao S, Wang Z, Hu Y and Xie B: Maternal lipids, BMI and IL17/IL35 imbalance in concurrent gestational diabetes mellitus and preeclampsia. Exp Ther Med 16: 427-435, 2018

30. Sun W, Cui B, Hong F and Xu Y: Establishment of ApoE-knockout mouse model of preeclampsia and relevant mechanisms. Exp Ther Med 12: 2634-2638, 2016.

31. Aouache R, Biquard L, Vaiman D and Miralles F: Oxidative stress in preeclampsia and placental diseases. Int J Mol Sci 19: 1496, 2018.

32. Staff AC, Dechend R and Redman CWG: Review: Preeclampsia, acute atherosis of the spiral arteries and future cardiovascular disease: Two new hypotheses. Placenta 34 (Suppl): S73-S78, 2013.

33. Dolnikoff M, Martín-Hidalgo A, Machado UF, Lima FB and Herrera E: Decreased lipolysis and enhanced glycerol and glucose utilization by adipose tissue prior to development of obesity in monosodium glutamate (MSG) treated-rats. Int J Obes Relat Metab Disord 25: 426-433, 2001.

34. Antwi E, Amoakoh-Coleman M, Vieira DL, Madhavaram S, Koram KA, Grobbee DE, Agyepong IA and Klipstein-Grobusch K: Systematic review of prediction models for gestational hypertension and preeclampsia. PLoS One 15: e230955, 2020

35. Hirschmugl B, Desoye G, Catalano P, Klymiuk I, Scharnagl H, Payr S, Kitzinger E, Schliefsteiner C, Lang U and Wadsack C: Maternal obesity modulates intracellular lipid turnover in the human term placenta. Int J Obes (Lond) 41: 317-323, 2017.

36. Brantsæter AL, Haugen M, Samuelsen SO, Torjusen H, Trogstad L, Alexander J, Magnus P and Meltzer HM: A dietary pattern characterized by high intake of vegetables, fruits, and vegetable oils is associated with reduced risk of preeclampsia in nulliparous pregnant norwegian women. J Nutr 139: 1162-1168, 2009.

37. Song T, Lu J, Deng Z, Xu T, Yang Y, Wei H, Li S, Jiang S and Peng J: Maternal obesity aggravates the abnormality of porcine placenta by increasing N6-methyladenosine. Int J Obes (Lond) 42: 1812-1820, 2018

38. Moyes AJ, Maldonado-Pérez D, Gray GA and Denison FC: Enhanced angiogenic capacity of human umbilical vein endothelial cells from women with preeclampsia. Reprod Sci 18: 374-382, 2011.

This work is licensed under a Creative Commons Attribution-NonCommercial-NoDerivatives 4.0 International (CC BY-NC-ND 4.0) License. 